\title{
Batch-wise Calibration of Multi-Gas-Sensors and Calibration Models for Mono Gas and Multi Gas Applications
}

\author{
Hubert B. Keller ${ }^{1}$, Rolf Seifert ${ }^{1}$ \\ ${ }^{1}$ Institute for Applied Informatics (IAI), Karlsruhe Institute of Technology (KIT) \\ Hermann-von-Helmholtz-Platz 1, D-76344 Eggenstein-Leopoldshafen, Germany \\ rolf.seifert@kit.edu
}

\begin{abstract}
:
Although sensor chips for multi-gas-sensors are usually batch-wise fabricated, each sensor chip has to be individually calibrated to yield a high analytic performance. For multi gas applications, a sensor chip normally has to be measured for calibration at about 5 calibration points for every component, $A$ new mathematical procedure for batch-wise calibration called ProCal was developed and extended to reduce the calibration effort nearly by a factor of five even for multi-gas-analysis. By application of the procedure to sensitivity data sampled at 5 mono gas applications and 4 multi component applications it is demonstrated that with this efficient calibration procedure the maximum analysis error for determination of the gas concentrations was estimated to less than $11 \%$ for multi-gas-applications.
\end{abstract}

Key words: multi-gas-sensors, batch-wise calibration, multi-gas- applications, multi-gas-analysis, analysis error

\section{Background \\ Economic online and in-situ field analyses applications like discriminated alarming of smouldering fire or toxic gas leakages, monitoring of volatile components in chemical and biochemical processes, quality monitoring in food processing etc., wait for reliable and economic analytical solutions by sensor systems.}

In this context, the isothermally operated metal oxide gas sensors (MOGs) with tin oxide as base material are manifold introduced due to their high sensitivity, long-term stability and low price. Their sensitivity to specific gas components, however, cannot be cultivated with high discrimination to others. Therefore, other approaches are necessary like a gas sensor array of MOGs [1,2] or by thermo cyclic operation of the MOG and simultaneous sampling of the conductance which finally leads to the mathematical analysis of the so called Conductance-over-Time-Profiles (CTPs) [3]. These CTPs show characteristic profile shapes reflecting gas composition and gas component concentrations depending on the choice of additives. It could be shown that especially using this approach, valuable signal information can be extracted to be numerically analyzed for substance identification and concentration determination even in the case of varying environmental conditions (e.g., humidity) [4].

The effort for calibration of the sensor elements is very time-consuming and costly. Sensor elements are usually batch-wise fabricated. But unavoidable production inaccuracies of the sensor elements lead to unreproducibilities of the gas analytic attributes. Therefore although the signal patterns of the various sensor elements of a production batch are quite similar, each sensor element has to be costly calibrated, in order to yield high analytic performance. The number of components to be simultaneously analyzed determines the dimension of the calibration field and for good analysis results, as was experimentally shown, about three to five or even more calibration points for every dimension are necessary depending on the accuracy demands of analysis.

\section{The Program ProCal}

To reduce the above mentioned immense effort for calibration a mathematical procedure for batch-wise calibration called ProCal was developed [5]. In the first version ProCal was designed for batch-wise calibration of mono gas applications. ProCal uses the fact that the 
signal patterns of sensor elements of a production line are different but anyway similar in their structure which can be seen in Fig. 1. ProCal is able to determine the so-called class reference chip of a batch and to determine outliers i.e. chips which cannot be calibrated with this procedure.

According to the basic idea of ProCal only the signal patterns of one single sensor chip (the class reference chip) of the batch are sampled at all calibration points (e.g., five calibration points for a mono-gas component analysis application or for each component of a multicomponent analysis application as well) of a calibration route. All the other chips of the batch are measured at only one calibration point (the so-called reference point). Next, for every sensor chip a mathematical function, the socalled approximation function, is determined which fits the signal pattern of the reference point, yield by the class reference chip, to the signal pattern yield by the corresponding sensor chip in a best manner. That means for each sensor chip excluding the class reference chip a sensor specific approximation function is obtained.

With these approximation functions the signal patterns of all chips (except the class reference chip) of the batch are numerically estimated at all the other calibration points using the measured signal patterns of the class reference chip at the corresponding concentrations. This means the signal patterns of nearly all calibration points (except of one) and nearly all chips (except of one) are approximated numerically.

Using this approach a calibration model is obtained called "batch-wise calibration model".

\section{Mathematical Calibration Models}

To compare the analysis accuracy three mathematical calibration models are established. The "individual model of each chip" means that each sensor element of a chip is individually calibrated. This is a very costly and time consuming calibration process and leads to the best possible analyses results. Using the "class reference model" only one sensor element of a batch (here the class reference chip) is completely calibrated and the calibration model of this chip is assigned to each other chip. This leads to very poor analysis results which can be seen in the following. The third calibration model is the above mentioned "batch-wise calibration model" with reduction of the complete calibration effort by a factor of 5 . Therefore and for batch-wise calibration of multi gas applications ProCal is extended. The data analysis is performed with the computer program ProSens [6].

\section{Applications}

Five mono gas applications (dimethyl sulfate (DMS), methyl, acetic acid, hexanal and octen, number of applications 1 - 5) and 4 multi gas applications (ternary acetic acid/hexanal/octenmixtures, number of applications 6 - 9) were considered and the gas probes were measured by periodic variation of the sensor temperature and simultaneous sampling of the so called Conductance-over-Time-Profiles (CTPs) [3]. The investigation was based on a batch of 10 sensor chips, called $\mathrm{S} 1$ and so on. The extended version of ProCal calculates the signal patterns which are necessary for the determination of the calibration model instead of costly and time consuming measuring.

Fig. 1 shows the CTPs of the 10 sensor chips measuring DMS (number of application: 1 ) at the concentration level 81200 which was chosen as reference point. It can be clearly seen that the CTPs are quite different but anyway similar in their structure. In this application ProCal determined sensor S4 as reference chip and no sensor chip had to be excluded from the batch-wise calibration procedure.

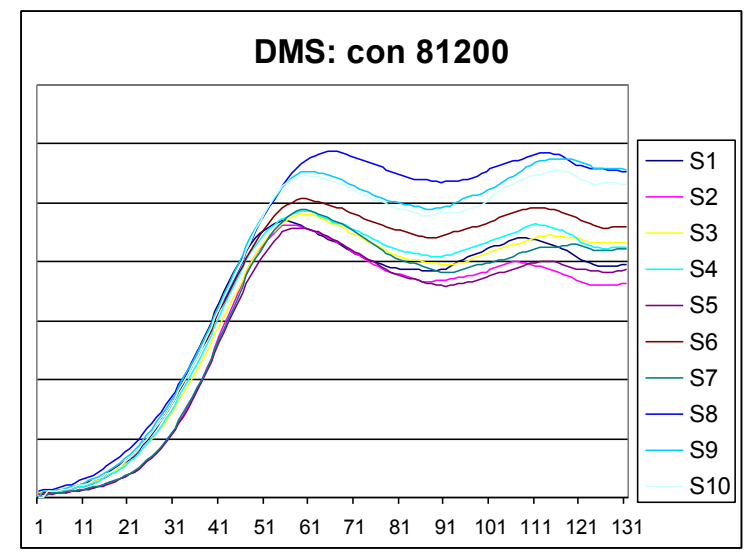

Fig. 1. CTPs of the 10 sensor chips measuring DMS at the concentration level 81200 (reference point)

In this application, Fig. 2 and Fig. 3 exemplary show for sensor S1 how close calculated and measured signal patterns are. That means that the calculated signal pattern can be used instead of the measured ones. This leads to a significant reduction of the costly and time consuming calibration process.

Fig. 4 shows the CTPs of the 10 sensor chips measuring a ternary acetic acid/hexanal/octenmixture (number of application: 6) at the concentration level 81200 which was again chosen as reference point. In this application 
sensor S10 was determined as reference chip and ProCal recognized the sensors S1, S3, S8 and $S 9$ as outliers which cannot be calibrated with this procedure.

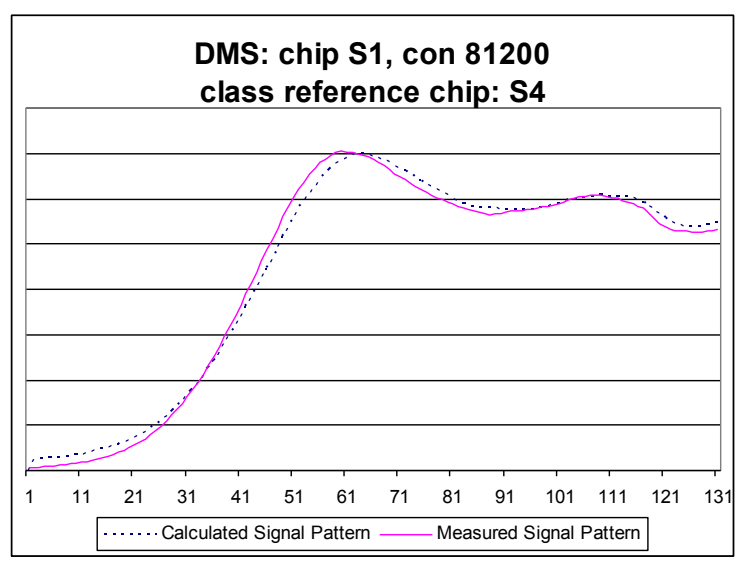

Fig. 2. DMS application: Comparison of calculated and measured signal pattern (CTP) of chip S1 at concentration level 81200 (reference point) using chip S4 as class reference chip.

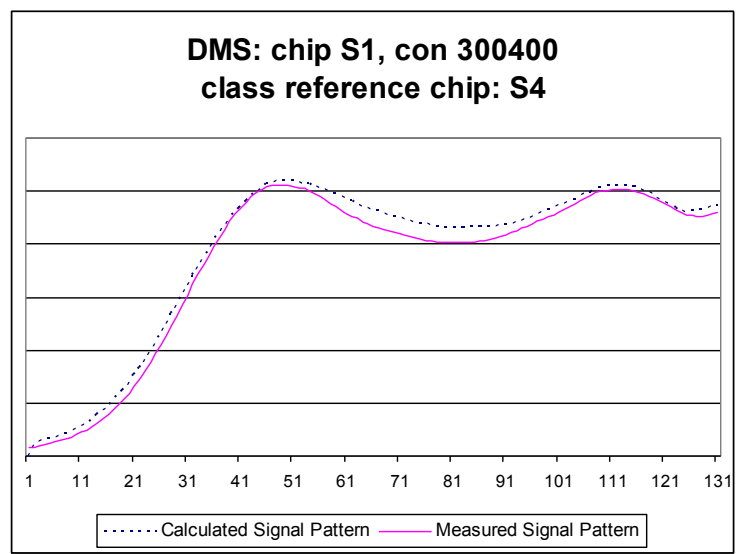

Fig. 3. DMS application: Comparison of calculated and measured signal pattern (CTP) of chip S1 at concentration level 300400 using chip S4 as class reference chip.

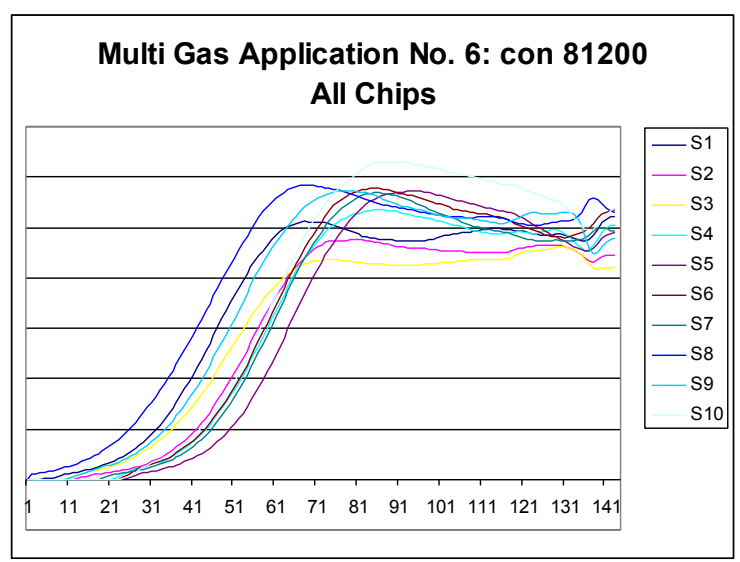

Fig. 4. CTPs of the 10 sensor chips measuring a ternary acetic acid/hexanal/octen-mixture (number of application 6) at the concentration level 81200 (reference point)
Fig. 5 shows the CTPs of the non-excluded sensor chips measuring the same ternary acetic acid/hexanal/octen-mixture.

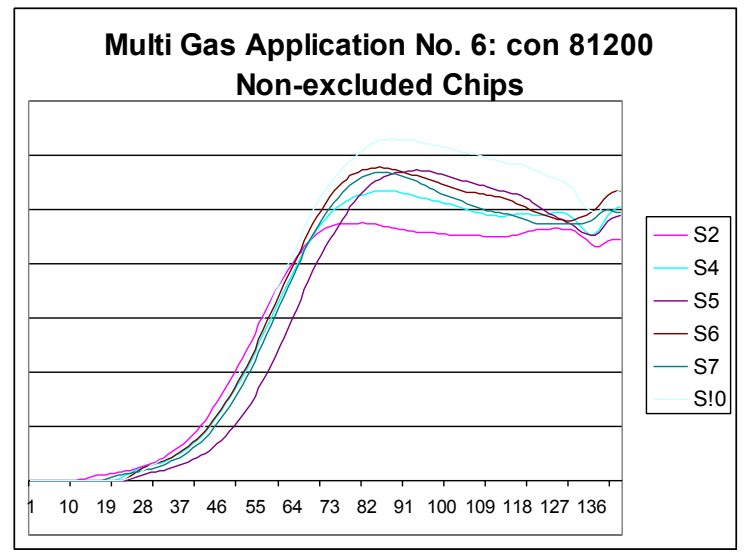

Fig. 5. CTPs of the non-excluded sensor chips measuring a ternary acetic acid/hexanal/octenmixture (number of application 6) at the concentration level 81200 (reference point)

The comparison of calculated CTP and measured CTP, exemplary shown for sensor S4 at concentration level 43500 in Fig. 6, demonstrates that also in this application the approximation is quite well.

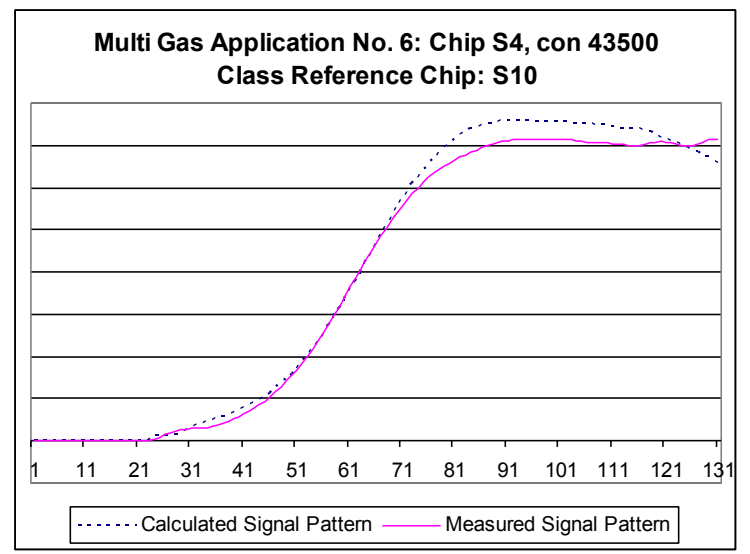

Fig. 6. Multi component application No. 6: Comparison of calculated and measured signal pattern (CTP) of chip S4 at concentration level 43500 using chip S10 as class reference chip.

To compare the analysis accuracy a data analyses on the basis of the above mentioned 3 calibration models was performed with the computer program ProSens.

In Fig. 7 the according maximum relative analysis results comparing dosed and estimated concentrations are listed over the 9 applications. The figure shows that the analysis results using batch-wise calibration are close to the best possible when using individual calibration, but the efforts for calibration are reduced by a factor of 5 . On the other hand they are drastically better than those which are based on class reference calibration. 


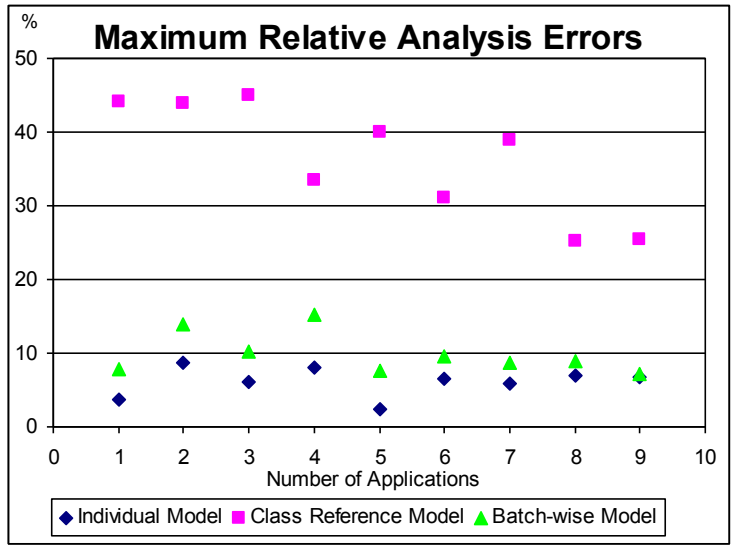

Fig. 7. Maximum relative analysis errors for the 9 application based on the 3 calibration models. Mono gas applications: 1-5, multi component applications: 6-9.

\section{Results}

Using the calibration procedure ProCal, the very time-consuming and expansive calibration of batch-wise fabricated sensor elements can be reduced almost by the factor of about five even in the case of multi-component applications. ProCal is able to determine the class reference chip, which represents the chips of the batch in a best manner and can exclude outliers, i.e chips which cannot be calibrated with this procedure. It could be shown that the calculated signal patterns are close to the sampled signal patterns and the analysis errors using this calibration model are very close to best possible ones using the very time-consuming individual model, but dramatically better than those using the class reference model.

The same procedure can be also applied for cost-effective and time-saving recalibration.

\section{References}

[1] P. Althainz, J. Goschnick, S. Ehrmann, and H. J. Ache, Sens. Actuators, B 33, 72 (1996), doi:10.1016/0925-4005(96)01838-2

[2] V. V. Sysoev, I. Kiselev, M. Frietsch, and J. Goschnick, Sensors 4, 37 (2004), doi:10.3390/ s40400037

[3] A. Jerger, H. Kohler, F. Becker, H.B. Keller, R. Seifert, Sensors and Actuators B, 81(2002) S.301-07; doi:10.1016/s0925-4005(01)00970-4

[4] K. Frank, V. Magapu, V. Schindler, H. Kohler, H. B. Keller, R. Seifert, Sensors Letters 6, 908 (2008); doi: 10.1166/sl.2008.527

[5] R. Seifert, H. B. Keller, K. Frank, H. Kohler, Sensor Letters 9, 621-624 (2011); doi:10.1166/ sl.2011.1576

[6] R. Seifert, H. B. Keller, K. Frank, H. Kohler, Sensor Letters 9, 7-10 (2011); doi:10.1166/ sl.2011.1408 